\title{
In situ permeation study of drug through the stratum corneum using attenuated total reflection Fourier transform infrared spectroscopic imaging
}

\author{
Jean-Michel Andanson \\ Imperial College London \\ Department of Chemical Engineering \\ South Kensington Campus \\ London SW7 2AZ \\ United Kingdom
}

\author{
Jonathan Hadgraft \\ University of London \\ The School of Pharmacy \\ 29-39 Brunswick Square \\ London WC1N 1AX \\ United Kingdom
}

\section{Sergei G. Kazarian}

Imperial College London

Department of Chemical Engineering

South Kensington Campus

London SW7 2AZ

United Kingdom

\begin{abstract}
Infrared (IR) spectroscopy is one of the most chemically specific analytical methods that gives information about composition, structure, and interactions in a material. IR spectroscopy has been successfully applied to study the permeation of xenobiotics through the skin. Combining IR spectroscopy with an IR array detector led to the development of Fourier transform infrared (FTIR) spectroscopic imaging, which generates chemical information from different areas of a sample at the microscopic level. This is particularly important for heterogeneous samples, such as skin. Attenuated total reflection (ATR)-FTIR imaging has been applied to measure, in situ, the diffusion of benzyl nicotinate (BN) through the outer layer of human skin [stratum corneum (SC)]. In vitro experiments have demonstrated the heterogeneous distribution of $\mathrm{SC}$ surface lipids before the penetration of a saturated solution of BN. Image analysis demonstrated a strong correlation between the distribution of lipids and drugs, while ethanol appeared to be homogenously distributed in the SC. These results show the ability of ATR-FTIR imaging to measure simultaneously the affinities of drug and solvent to the lipid-rich and lipid-poor skin domains, respectively, during permeation. This information may be useful in better understanding drug-diffusion pathways through the SC. $\odot 2009$ Society of Photo-Optical Instrumentation Engineers. [DOI: 10.1117/1.3130324]
\end{abstract}

Keywords: diffusion; drug; human skin; stratum corneum; imaging; attenuated total reflection; Fourier transform infrared spectroscopy; FT-IR.

Paper 08443R received Dec. 15, 2008; revised manuscript received Mar. 13, 2009 ; accepted for publication Mar. 17, 2009; published online May 12, 2009; corrected May 19, 2009.

\section{Introduction}

Human skin is a naturally efficient barrier that protects the body from physical and chemical "onslaught." The first layer of our skin is a barrier to most molecules; nevertheless, the skin can be used as a route to deliver drugs systemically. But despite several decades of intensive research, the precise mechanism of dermal absorption is still unclear. The stratum corneum (SC), which is the outer skin layer with a thickness of $\sim 10$ to $20 \mu \mathrm{m}$, is a structure of dead and anucleate cells (corneocytes) surrounded by a lipid matrix that can be visually described as a "brick and mortar" model. Molecules can use an intracellular pathway (the "brick") and/or intercellular pathway (the "mortar") to diffuse through the $\mathrm{SC}^{2-4}$ The lipid bilayers surrounding the SC cells provide a key way of controlling the drug flux. The organization of the lipid matrix may be sensitive to any perturbation, such as temperature. ${ }^{5,6}$ or chemical absorption.

The interaction between a molecule and the different parts of the SC may play a role in determining which route a drug

Address all correspondence to: Sergei Kazarian, Chemical Engineering, Imperial College London, South Kensington Campus-London, SW7 2AZ United Kingdom; Tel: 44-207-594-5574; Fax: 44-207-594-1989; E-mail: s.kazarian@imperial.ac.uk will use to pass the first skin layer or make the SC almost impermeable to the component. Different penetration enhancers have been developed to improve the delivery of drugs via the skin $;{ }^{8}$ the presence of the enhancer affects drug penetration. However, our understanding of the underlying chemistry involved during drug diffusion through the SC still needs to be improved.

Infrared (IR) spectroscopy is one technique that has been developed to study drug diffusion through the skin. ${ }^{9,10}$ It is also able to measure humidity variations ${ }^{11}$ and the structure of lipid bilayers. ${ }^{12}$ In this context, Fourier transform infrared (FTIR) spectroscopic imaging was applied to skin to measure the heterogeneity of the skin at a microscopic level. ${ }^{6,12-20}$ More details on imaging using vibrational spectroscopy and some examples of biomedical applications can be found in Refs. 6 and 13-25. Using FTIR and/or confocal Raman microscopy, Mendelsohn et al. were able to observe the penetration of several exogenous molecules in a transverse section of skin. ${ }^{26,27}$ FTIR imaging also has been used to measure the heterogeneity of the SC structure. ${ }^{28}$ More recently, Jiang et al. studied porcine skin tissue to observe the diffusion of DMSO.$^{29}$ In that study, the tissue was sectioned and several

1083-3668/2009/14(3)/034011/8/\$25.00 @ 2009 SPIE 
skin layers were observed simultaneously. Another approach images one tissue layer by placing the skin on the surface of an attenuated total reflection (ATR) crystal and measuring permeation through the skin-crystal interface. Since the SC is the skin layer that restricts absorption, it is interesting to study how the drug and vehicles diffuse into and through this specific layer.

ATR-FTIR imaging was recently employed to study the skin, ${ }^{30}$ especially the behavior of SC under a broad range of humidities, and to demonstrate ATR-FTIR's use in studying transdermal drug delivery. ${ }^{11,31}$ This study demonstrated the possibility of measuring SC heterogeneity by observing lipidrich and lipid-poor regions, and the permeation and distribution of a model drug and solvents into the SC. Although this spectroscopic approach measures permeation through the skin, it is not a straightforward method for the accurate calculation of diffusion coefficients because the ATR crystal is present on one side of the skin instead of in an infinite sink. Thus, the diffusing material may form "pools" between the skin and the ATR crystal rather than being removed, as would happen in a true diffusion cell. To obtain a diffusion coefficient, it is possible to apply a Franz-type cell, as was used by Hartmann et al., ${ }^{9}$ in conventional ATR spectroscopic studies or in combination with a tape strip in ATR-FTIR imaging. ${ }^{31}$ The ATR-FTIR imaging approach presented here studies permeation of a solvent and model drug through the skin.

The choice of drug for this study was benzyl nicotinate $(\mathrm{BN})$. The transdermal diffusion of $\mathrm{BN}$ has already been studied by measuring the increase in skin oxygenation using electron paramagnetic resonance $(\mathrm{EPR})^{32}$ or laser Doppler flowmetry. ${ }^{33} \mathrm{BN}$ was chosen because it is a small lipophilic molecule $(\operatorname{cog} \mathrm{P}=2.66)$ that will diffuse mainly through the intercellular route of the SC.

\section{Materials and Methods}

\subsection{Materials}

Abdominal cadaver skin was obtained with appropriate ethical approval and prepared using a normal procedure. ${ }^{34} \mathrm{BN}$ (98\% pure) and PEG 400 were obtained from Sigma-Aldrich, UK. Silicone grease was used to seal the system during the diffusion process.

\subsection{FTIR Spectroscopic Imaging}

FTIR imaging is able to measure 4096 spectra simultaneously using a focal plane array (FPA) detector with $64 \times 64$ pixels. Each spectrum corresponds to a specific position on the surface of the ATR crystal (diamond). By plotting the integrated absorbance of a specific spectral band in the mid-IR spectrum, it is possible to obtain the distribution and amount of a particular compound in the different parts of the imaged area. FTIR imaging has been explained in detail in previous publications. ${ }^{16,35}$ A Varian imaging system was coupled with a Bio-Rad 60A FTIR spectrometer and an ATR-FTIR Golden Gate $^{\mathrm{TM}}$ accessory (Specac Ltd., UK) positioned in the large sample compartment. The total image size of this system was $550 \times 600 \mu \mathrm{m}^{2}$ with a pixel size resolution of approximately $9 \times 9 \mu \mathrm{m}^{2}$. Each spectrum was recorded with a resolution of $4 \mathrm{~cm}^{-1}$ and 64 co-additions (which took less than three minutes) between 4000 and $875 \mathrm{~cm}^{-1}$. The images were created using Varian Resolution Pro ${ }^{\mathrm{TM}} 4.0$ software. During the ex-

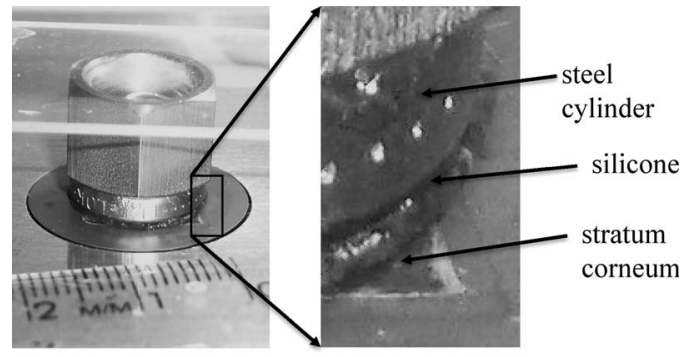

(a)

(b)

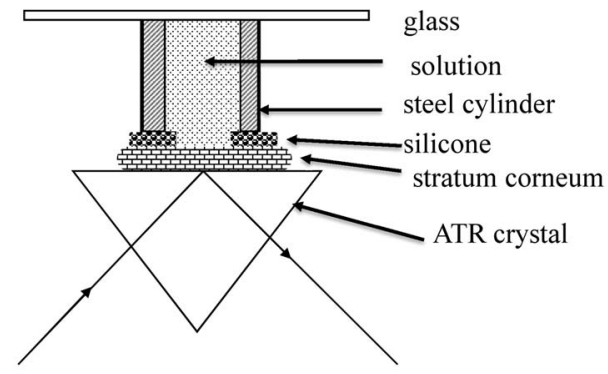

(c)

Fig. 1 Pictures (a) and (b) and schematic (c) of the equipment used for transversal diffusion through the SC on a Golden Gate ${ }^{T M}$ accessory.

periment, an image was recorded every 10 minutes during the first 2 hours, then every 20 minutes for the next 2 hours, and finally every hour for the next 6 hours.

\subsection{Skin Preparation}

Sample preparation involved cutting a $1 \mathrm{~cm}^{2}$ piece of SC (stored at $-20^{\circ} \mathrm{C}$ ), which was then defrosted to room temperature and rehydrated by leaving the sample on top of a drop of water (with the internal part of the skin in contact with the water). After an hour, the piece of skin was cleaned gently with ethanol and put back on top of adrop of water for a few minutes. Then a second drop of water was placed on the crystal of the ATR accessory and the SC positioned on top of the water drop (with the external part of the skin in contact with the water). Most of the excess liquid water was then carefully removed using tissue paper. The rest of the water evaporated naturally at room temperature in less than an hour. After this, the external side of the SC was adhered to the surface of the diamond. A first measurement was taken and showed nonhomogeneous skin contact with the diamond. It was possible to obtain homogenous contact by pressing quite hard on the SC. A stainless steel hollow cylinder of approximately $1 \mathrm{~cm}$ thickness with some silicone grease on the bottom was placed carefully on the dry SC. The grease was used to ensure contact between the cylinder and the SC to avoid any leakage. To rehydrate the SC, the cylinder was filled with deionized water. Figure 1 shows pictures and schematic of the equipment. The diffusion of the water into the dry SC took less than half an hour, which was observed by monitoring the absorbance of the bending mode of the water. During this time, several images were taken that demonstrated the lipid-rich areas in contact with the crystal are increasing during the skin rehydration. This effect may have resulted from the swelling of the skin; the skin surface was not completely flat, and the skin irregularities corresponded to the lipid region. When the skin 


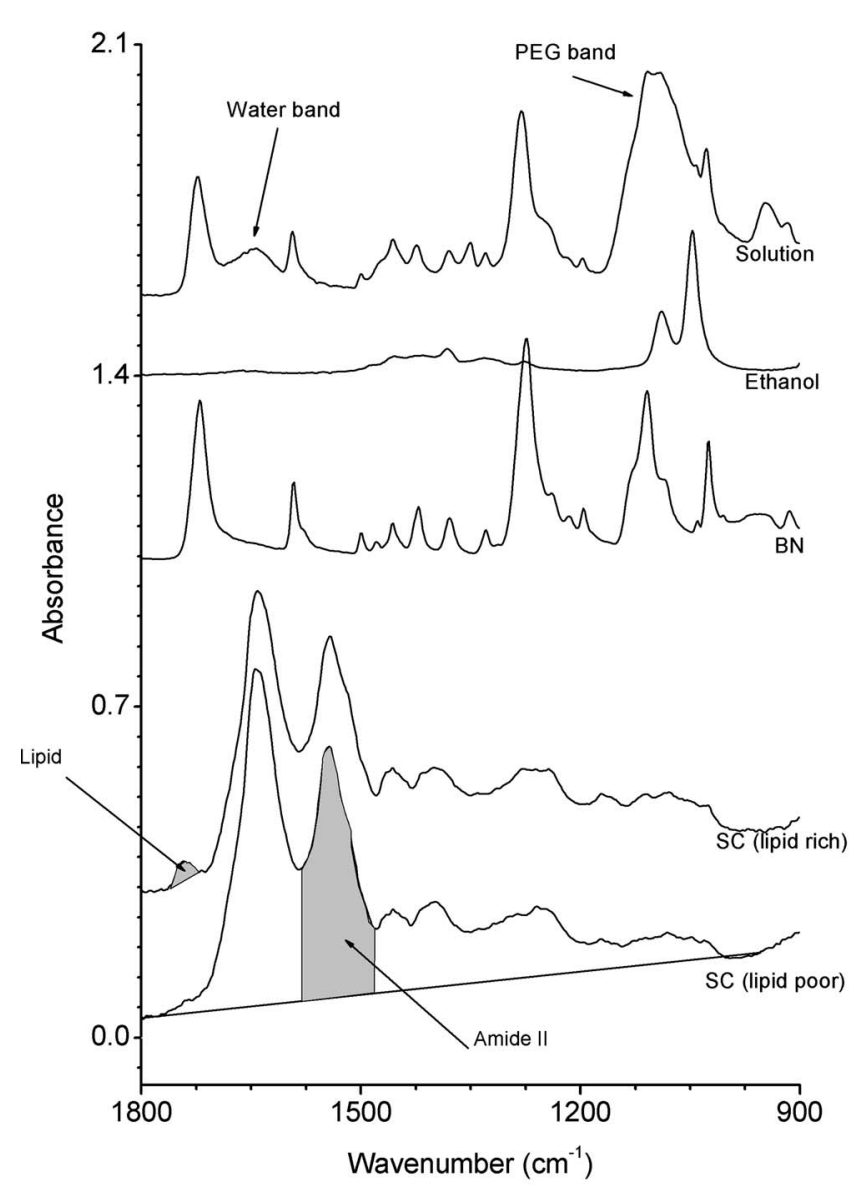

(a)

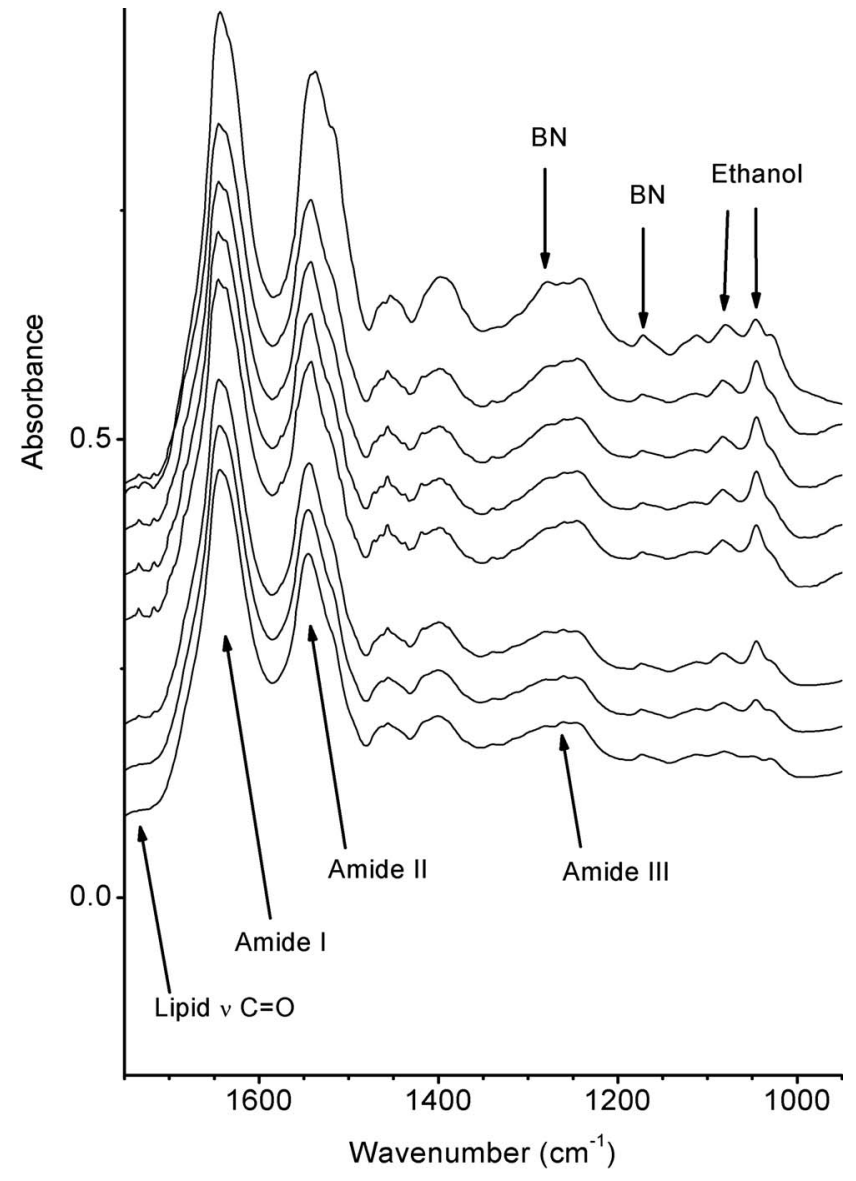

(b)

Fig. 2 (a) ATR-FTIR spectra of SC bands (lipid rich and lipid poor), BN, ethanol, and the solution. (b) Evolution of the spectra (average of the entire image) during the solution diffusion into the SC at $t=0,20,40,60,80,100,120$, and 240 minutes (from bottom to top).

swelled due to water absorption, the cavities may have been pushed down to the surface and brought into contact with the crystal, which increased the overall signal of the lipids.

The drug diffusion through the SC followed. The water in the cylinder was removed and replaced by a biphasic liquid with $70 \%$ (mass) water, 10\% PEG, $10 \%$ ethanol, and around $10 \%$ of BN (to achieve saturation). The solution was prepared 24 hours in advance and was chosen to keep the skin humidity constant by having water on the top of the skin. PEG is widely used in skin creams and should not diffuse into the SC, ${ }^{36}$ while ethanol should diffuse fast through the SC and may act as a penetration enhancer for the drug. ${ }^{37-39}$ It is also possible that the ethanol can extract some of the lipids.

In this condition, the excess BN (yellowish liquid) moved slowly to the bottom of the solution (density $1.165 \mathrm{~g} \mathrm{~cm}^{-3}$ ); therefore, the top solution (saturated with $\mathrm{BN}$ ) was removed and used in the diffusion experiments.

\section{Results and Discussion}

To follow the diffusion of the drug, three bands of BN that did not overlap strongly with the spectral bands of other components of the system were chosen. At the same time, IR bands of PEG and ethanol were selected to measure the evolution of their concentrations across the SC. In a complex system (BN, PEG, water, ethanol, and SC), many bands overlap, so the choice of the integration range for spectral bands to generate images can be crucial. For example, the amide I band is generally used to observe proteins even if the water bending mode is largely overlapping with this band. The spectra of the skin, BN, ethanol, and the solution are shown in Fig. 2. The amide I and II bands strongly overlapped with other bands, so we chose to integrate these two bands using a baseline between 1800 and $950 \mathrm{~cm}^{-1}$. For weaker bands, a different integration approach was applied. Examples of the amide II (using baseline) and lipid band (without baseline) integration are shown in Fig. 2(a).

One of the BN bands overlapped with the lipid band at $1740 \mathrm{~cm}^{-1}$. Consequently, it was not possible to follow the evolution of the lipid band using this band. The three strongest bands in the mid-IR of the BN were centered on 1020, 1110 , and $1260 \mathrm{~cm}^{-1}$. These three bands were used to measure the drug diffusion across the SC. For ethanol only, the strongest band at $1045 \mathrm{~cm}^{-1}$ was used. The evolution of the average spectra obtained by the 4096 pixels of the detector during the diffusion of the solution is illustrated in Fig. 2(b). The diffusion of ethanol was observable, while the diffusion of BN seemed to be slower. Finally, the averaged spectrum from all of the measured area did not clearly show the lipid band at $1740 \mathrm{~cm}^{-1}$, while the spectrum extracted from the pixel corresponding to the lipid-rich area shown in Fig. 2(b) 
demonstrated the ability of ATR-FTIR imaging to detect a localized compound even in a low concentration.

\subsection{Advantages and Limitations of Imaging Compared with Single-Element Detector}

ATR-FTIR imaging avoids some problems, such as the detection of a paper fiber left on the surface of the SC, which would be almost impossible to do with a single-element detector. The homogeneity of the contact (or lack of it) is also much easier to observe using an imaging approach than with a single-element detector, which does not provide the average spectrum information from the whole area. The contact between the crystal and the sample is crucial in ATR measurements because the IR beam probes a depth of approximately $1 \mu \mathrm{m}\left(2 \mu \mathrm{m}\right.$ at $1000 \mathrm{~cm}^{-1}$ and $0.5 \mu \mathrm{m}$ at $4000 \mathrm{~cm}^{-1}$ in the case of a diamond crystal and skin sample). ATR-FTIR imaging has a substantial advantage over a single-element detector because imaging allows us to control the contact and the homogeneity of the sample with the crystal. The advantages of using a FPA detector over a single-element detector for the sensitive detection of heterogeneously distributed substances on the skin surface have been demonstrated previously. ${ }^{16,31}$

Because of some chromatic aberrations caused by this particular setup, it was not possible to obtain the optimal focus on the skin surface at all wavenumbers of the IR spectra simultaneously. We chose to obtain a very sharp image in the fingerprint region where the focus was very good. With this focus, it is not possible to obtain well-focused images based on the distribution of the $\mathrm{OH}$ or $\mathrm{CH}$ stretching bands. For that reason, the $\mathrm{OH}$ stretching band is not used to follow the amount of water in the $\mathrm{SC}$, and the $\mathrm{CH}$ stretching was not used here to observe the distribution of the lipid-rich regions. Moreover, the detector cannot measure the IR spectrum below $875 \mathrm{~cm}^{-1}$. Consequently, all images presented in this article are based on the spectral bands in the 1800 to $900 \mathrm{~cm}^{-1}$ range. The main bands observed for SC, PEG, ethanol, water, and $\mathrm{BN}$ in the IR and the integration ranges used to obtain the images are summarized in Table 1 .

\subsection{Imaging of the $S C$}

To analyze the diffusion of a solution through the SC, it is important to ensure that the skin contact does not change during the process. It is possible to monitor the quality of the skin contact with the ATR crystal by following the images of the amide bands.

Figure 3 presents examples of images obtained using different spectral bands in one of the several reproducible experiments. The images of amide I and II show a variation in the contact; the small blue areas in the four images (amide I and II, before and after diffusion) are the positions where the amide bands were weaker, which corresponded to poor a contact between the skin and the diamond. The positions and the areas of these regions barely changed during the few hours of the experiment, which demonstrates that the SC did not change or move to a large extent during the experiment. The $\mathrm{C}=\mathrm{O}$ stretching lipid band (at $1740 \mathrm{~cm}^{-1}$ ) allowed us to plot the distribution of the lipid-rich areas on the SC surface before the diffusion of the solution. Since the BN also had a band at around $1720 \mathrm{~cm}^{-1}$, it was not possible to use this band to plot the distribution of the lipid-rich areas after diffu-
Table 1 Spectral assignment of single spectra from the imaging dataset and integration range for spectral bands of SC, BN, PEG, and ethanol.

\begin{tabular}{ccc}
\hline Vibration band & Integration range & $\begin{array}{c}\text { Baseline of } \\
\text { integration }\end{array}$ \\
\hline $1740 \mathrm{~cm}^{-1}$ Lipid $\nu_{(\mathrm{C}=0)}$ & $1760-1720$ & \\
$1640 \mathrm{~cm}^{-1}$ Amid I $\nu_{(\mathrm{C}=0)}$ & $1680-1590$ & $1800-950$ \\
$1540 \mathrm{~cm}^{-1}$ Amid II $\delta_{(\mathrm{NH})}+\nu_{(\mathrm{CN})}$ & $1590-1490$ & $1800-950$ \\
$1260 \mathrm{~cm}^{-1}$ & $1270-1250$ & \\
$1235 \mathrm{~cm}^{-1}$ Amid III & $1250-1220$ & $1280-1220$ \\
$1280 \mathrm{~cm}^{-1}$ BN band & $1300-1265$ & \\
$1110 \mathrm{~cm}^{-1}$ BN band & $1120-1100$ & \\
$1020 \mathrm{~cm}^{-1}$ BN band & $1040-1005$ & \\
$1100 \mathrm{~cm}^{-1}$ PEG band & $1200-970$ & \\
$1045 \mathrm{~cm}^{-1}$ Ethanol band & $1065-1035$ & \\
\hline
\end{tabular}

sion. The diffusion did not change the amide I and II images, which was not the case for the lipid band at the $1740 \mathrm{~cm}^{-1}$ band.

The images obtained using the bands of the different components of the solution are shown in Fig. 4. For the BN, three bands were used to observe its distribution. The absorbance of the three bands increased, and the BN distribution after diffusion was heterogeneous for the three images. The shapes of domains in these images are not identical, but considering the relatively weak absorbance of the bands and the overlap with the skin bands, the three bands produced a similar map. It was not possible to obtain a perfect correlation between images even when images from the same compound were compared.

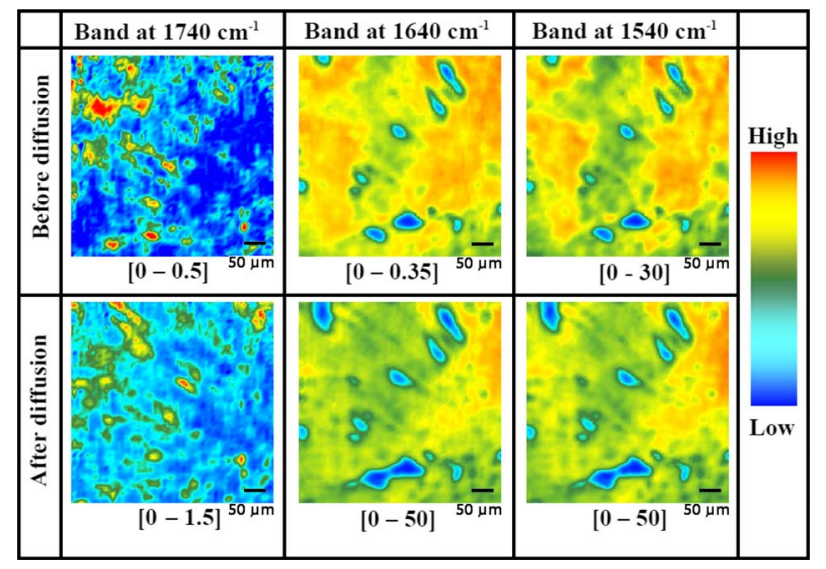

Fig. 3 ATR-FTIR images of SC bands before and after the diffusion. The labels for bands at 1740,1640 , and $1540 \mathrm{~cm}^{-1}$ correspond to the lipid $\mathrm{C}=\mathrm{O}$ stretching band centered at $1740 \mathrm{~cm}^{-1}$, the amide I band centered at $1640 \mathrm{~cm}^{-1}$ and the amide II band centered at $1540 \mathrm{~cm}^{-1}$, respectively. The values in brackets correspond to the color bar value, with the minimum in blue and maximum in red. (Color online only.) 


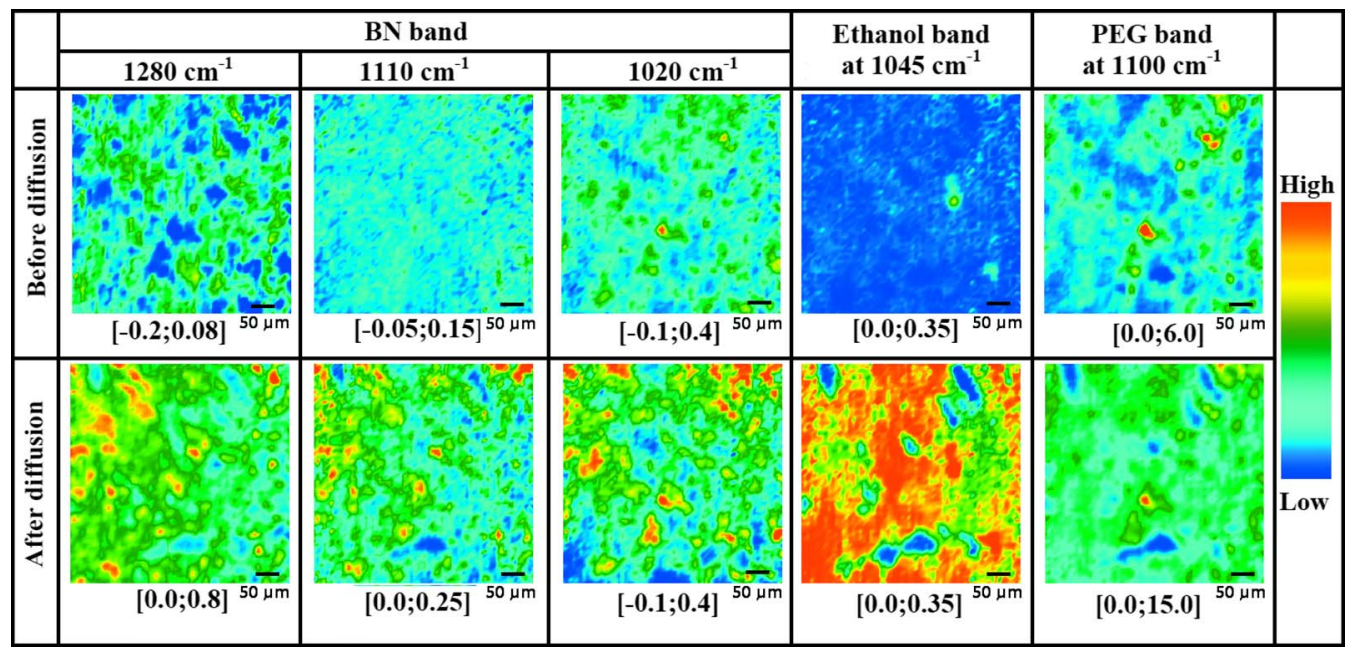

Fig. 4 ATR-FTIR images based on the bands of BN, ethanol, and PEG before and after the solution diffusion.

For example, the value of the integration at $1020 \mathrm{~cm}^{-1}$ was the result of a band from the skin and a band from the $\mathrm{BN}$, which were both in the same range.

In another example, the band at $1280 \mathrm{~cm}^{-1}$ was the strongest band of $\mathrm{BN}$ in the mid-IR. Even if skin does not have a strong band at this wavenumber, the presence of amide III in the same range can slightly change the value of the integration and can also modify, to some extent, the shape of the image. Nevertheless, in the images before and after BN diffusion (Figs. 3 and 4) there is a fair correlation between the position of the lipid-rich areas before the diffusion and the position of the BN after it crossed the SC.

The images built using the spectral band of ethanol clearly show a diffusion of the solvent through the SC. The image of the ethanol distribution after diffusion almost coincides with the image based on the amide I and II bands. The ethanol was homogenously distributed in the SC, at least for the $10-\mu \mathrm{m}$ resolution used here.

Finally, the images based on the distribution of the PEG band show that the integration band used for PEG increased during the diffusion. Nevertheless, since this integration is rather broad, the evolution of the absorbance of this band may not necessarily be showing that the PEG was diffusing. Other bands, like ethanol at $1045 \mathrm{~cm}^{-1}$ or $\mathrm{BN}$ at 1110 and $1020 \mathrm{~cm}^{-1}$, overlapped with the PEG band and increased during the process, which could partly explain the effect of the increasing domain size. It is not possible from this analysis to conclude that PEG diffused through the SC.

Another way to view the correlation between the spatial concentrations of two species in the FTIR images is to compare directly the absorbance of two bands from spectra extracted from each pixel. Figures 5(a) and 5(b), respectively, illustrate the correlation of the lipid band $\left(1740 \mathrm{~cm}^{-1}\right)$ before and after diffusion and the correlation between the lipid band before and the BN band after diffusion. The drug and lipid bands are only moderately correlated, but the lipid band is also not perfectly self-correlated as a function of time. The evolution of the lipid-rich area with time can be explained by the presence of ethanol in the SC. ${ }^{39,40}$

To overview the correlation between different bands, a correlation matrix is shown in Tables 2 and 3. Table 2 contains the self-correlations and correlations between the lipid, amide, $\mathrm{BN}$, and ethanol bands after diffusion. Table 3 shows the correlations between these bands before (amide and lipid) and after (amide, lipid, BN, and ethanol bands) penetration. A perfect correlation between two images is 1 ; when two images were not correlated at all, the value is zero; and when two images were negative to each other, the value is -1 . Table 3 does not give correlations for $\mathrm{BN}$ and ethanol before diffusion because these species were not present. From both tables, it

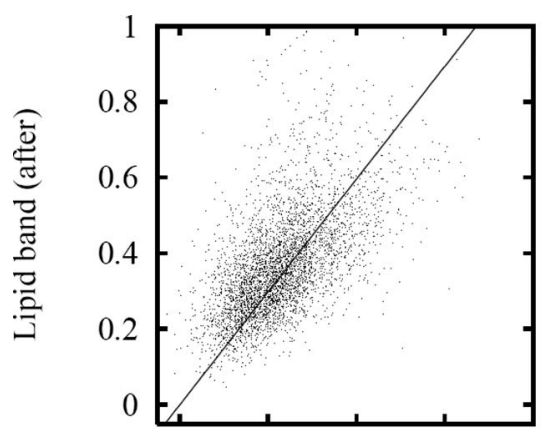

(a)

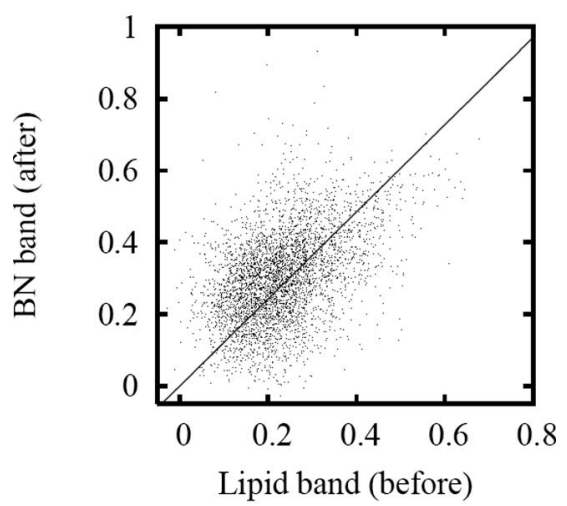

(b)

Fig. 5 Correlation of lipid and $\mathrm{BN}$ bands using the spectra of each pixel showing the absorbance of the lipid band at $1740 \mathrm{~cm}^{-1}$ (A) before and after the diffusion, and (B) before the diffusion versus the absorbance of the $\mathrm{BN}$ band at $1280 \mathrm{~cm}^{-1}$. 
Table 2 Correlation matrix between main spectral bands after diffusion.

\begin{tabular}{|c|c|c|c|c|c|c|c|}
\hline & $1280 \mathrm{BN}$ & 1110 & $\mathrm{BN}$ & Lipid & Amide I & Amide II & Ethanol \\
\hline $1280 \mathrm{BN}$ & 1 & & & & & & \\
\hline $1110 \mathrm{BN}$ & 0.635 & 1 & & & & & \\
\hline Lipid & 0.655 & 0.7 & & 1 & & & \\
\hline Amide I & 0.029 & 0.0 & & -0.077 & 1 & & \\
\hline Amidell & 0.003 & -0.0 & & -0.109 & 0.880 & 1 & \\
\hline Ethanol & 0.139 & 0.1 & & 0.021 & 0.583 & 0.696 & 1 \\
\hline
\end{tabular}

appears that the lipid $\left(1740 \mathrm{~cm}^{-1}\right)$ and drug $\left(1280 \mathrm{~cm}^{-1}\right.$ and $1110 \mathrm{~cm}^{-1}$ ) bands were correlated and present in the same areas of the images, and in similar concentrations. The amide (1540 and $\left.1640 \mathrm{~cm}^{-1}\right)$ and ethanol $\left(1045 \mathrm{~cm}^{-1}\right)$ bands can be grouped in a second cluster; the correlation between these two groups is nearly nonexistent. By considering these correlation tables, it is clear that the distribution of the drug was correlated to the lipid-rich area. On the other hand, the concentration of the ethanol was distributed more homogenously. A strong correlation cannot be observed between the ethanol and proteins (based on the amide bands) because these species were distributed relatively homogenously in the images. Since the lipid-rich areas are quite small in these images, a strong anti-correlation cannot be obsereved between the lipid-rich and protein-rich areas. Finally, the relatively small correlation, around 0.5 , between the two drug bands after diffusion and the lipid band before diffusion (Table 3, 3rd line) can be explained by the fact that these bands were relatively weak, which decreased the signal-to-noise ratio. In addition, the lipid-rich areas were probably slightly affected during the diffusion by the presence of ethanol.

The contrast between the distributions of $\mathrm{BN}$ and ethanol illustrates very well the difference in distribution. Even if it is not possible to measure directly the spectrum of the lipid bilayers between the cells, which is too small to be assessed with a resolution of a few microns, it is possible to measure the heterogeneity of the lipid distribution and to correlate it with the drug distribution. This is the first time that such a correlation has been demonstrated by the use of FTIR spectroscopic imaging. This study should shed light into the mechanisms of drug permeation from complex topical formulations.

Beyond the analysis of single images, the ensemble of data can be used to provide information about the kinetics of ethanol and $\mathrm{BN}$ permeation into the SC. The evolution of the absorbance of the bands extracted from the spectra of one arbitrary pixel in an image is shown in Fig. 6. The ethanol diffused quickly through the SC, and the ethanol concentration reached its maximum after 100 minutes and decreased slowly after that. The amount of ethanol may have decreased slightly in the solution on the top of the skin due to evaporation, and because of the existence of an equilibrium between the ethanol in the SC and in the solution.

In contrast, the maximum concentration of $\mathrm{BN}$ in the $\mathrm{SC}$ was reached after 200 minutes and then the concentration stayed approximately constant. The intensities of specific bands of $\mathrm{BN}$ and ethanol show the evolution of the concentration of these species during the diffusion process, while an analysis of an image shows the homogeneity/heterogeneity of the system at the skin surface.

\section{Conclusions}

A solution of $\mathrm{BN}$ in a mixture of ethanol, water, and PEG was used to study permeation in vitro across a human skin sample.

Table 3 Correlation matrix between main bands before and after diffusion. Since there is no drug or ethanol before diffusion, the lines corresponding to these data are empty.

\begin{tabular}{ccccccc}
\hline $\begin{array}{c}\text { After } \\
\text { Before }\end{array}$ & $1280 \mathrm{BN}$ & $1110 \mathrm{BN}$ & Lipid & Amide I & Amide II & Ethanol \\
\hline $1280 \mathrm{BN}$ & - & - & - & - & - & - \\
$1110 \mathrm{BN}$ & - & - & - & - & - & - \\
Lipid & 0.439 & 0.516 & 0.572 & 0.023 & 0.041 & -0.075 \\
Amide I & -0.005 & -0.051 & -0.177 & 0.854 & 0.747 & 0.347 \\
Amidell & -0.075 & -0.051 & -0.185 & 0.749 & 0.745 & 0.264 \\
Ethanol & - & - & - & - & - & - \\
\hline
\end{tabular}




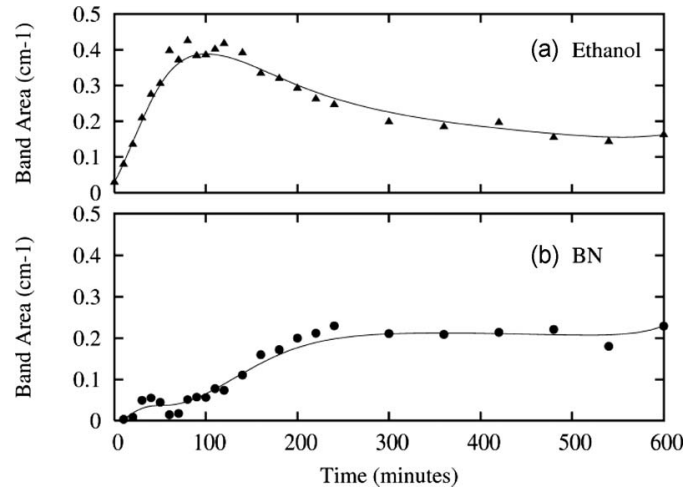

Fig. 6 Evolution of the absorbance of the (a) BN band $\left(1280 \mathrm{~cm}^{-1}\right)$ and (b) ethanol band $\left(1045 \mathrm{~cm}^{-1}\right)$ extracted from one pixel during the diffusion process.

The in situ measurement of the permeation kinetics of BN and ethanol through the SC was achieved using ATR-FTIR imaging spectroscopy. The FTIR images obtained during and after the permeation experiments clearly show that the ethanol and BN were distributed differently in the SC. The spatial resolution used in this approach was sufficient to image the heterogeneous distribution of lipids. The distribution of the BN mainly coincided with the distribution of the lipid-rich areas in the SC, while the distribution of ethanol correlated well with the distribution of the amide band in the SC.

This is the first time that an in situ experiment using ATRFTIR imaging on human skin has demonstrated the potential of this technique to observe the distribution heterogeneity of different compounds diffusing through the SC. The FTIR spectra corresponding to specific locations in the SC provided information about the evolution of the concentration of the drug and solvent during penetration.

\section{Acknowledgments}

We thank the Engineering and Physical Sciences Research Council (EP/D033284/1 \& EP/D50273X/1) for financial support. We also thank Dr. M. Lane, Dr. J. Tetteh, Dr. W. J. McAuley, and Ms. K. T. Mader for their help and advice.

\section{References}

1. A. Williams, Transdermal and Topical Drug Delivery, Pharmaceutical Press, London (2003).

2. P. S. Talreja, G. B. Kasting, N. K. Kleene, W. L. Pickens, and T. F. Wang, "Visualization of the lipid barrier and measurement of lipid pathlength in human stratum corneum," AAPS PharmSci 3(2), 48-56 (2001).

3. B. Illel, H. Schaefer, J. Wepierre, and O. Doucet, "Follicles play an important role in percutaneous absorption," J. Pharm. Sci. 80, 424 427 (1991)

4. J. Lademann, N. Otberg, H. Richter, U. Jacobi, H. Schaefer, U. Blume-Peytavi, and W. Sterry, "Follicular penetration. An important pathway for topically applied substances," Der Hautarzt 54, 321-323 (2003).

5. C. L. Gay, R. H. Guy, G. M. Golden, V. H. Mak, and M. L. Francoeur, "Characterization of low-temperature (i.e., $<65$ degrees $\mathrm{C}$ ) lipid transitions in human stratum corneum," J. Invest. Dermatol. 103, 233-239 (1994)

6. R. Mendelsohn and M. Diem, "Vibrational microscopic imaging," Biochim. Biophys. Acta Biomembr. 1758, 813-813 (2006).

7. A. N. C. Anigbogu, A. C. Williams, B. W. Barry, and H. G. M. Edwards, "Fourier transform raman spectroscopy of interactions between the penetration enhancer dimethyl sulfoxide and human stra- tum corneum," Int. J. Pharm. 125, 265 (1995).

8. H. A. E. Benson, "Transdermal drug delivery: penetration enhancement techniques," Curr. Drug Deliv. 2, 23-33 (2005).

9. M. Hartmann, B. D. Hanh, H. Podhaisky, J. Wensch, J. Bodzenta, S. Wartewig, and R. H. H. Neubert, "A new FTIR-ATR cell for drug diffusion studies," Analyst (Cambridge, U.K.) 129, 902-905 (2004).

10. E. Touitou, V. M. Meidan, and E. Horwitz, "Methods for quantitative determination of drug localized in the skin," J. Controlled Release 56, 7-21 (1998).

11. K. L. A. Chan and S. G. Kazarian, "Chemical imaging of the stratum corneum under controlled humidity with the attenuated total reflection Fourier transform infrared spectroscopy method," J. Biomed. Opt. 12, 044010-044010 (2007).

12. R. Mendelsohn, C. R. Flach, and D. J. Moore, "Determination of molecular conformation and permeation in skin via IR spectroscopy, microscopy, and imaging," Biochim. Biophys. Acta Biomembr. 1758 , 923-933 (2006).

13. H. Fabian, N. A. N. Thi, M. Eiden, P. Lasch, J. Schmitt, and D. Naumann, "Diagnosing benign and malignant lesions in breast tissue sections by using IR-microspectroscopy," Biochim. Biophys. Acta Biomembr. 1758, 874-882 (2006).

14. R. Bhargava, D. C. Fernandez, S. M. Hewitt, and I. W. Levin, "High throughput assessment of cells and tissues: Bayesian classification of spectral metrics from infrared vibrational spectroscopic imaging data," Biochim. Biophys. Acta Biomembr. 1758, 830-845 (2006).

15. A. Kretlow, Q. Wang, J. Kneipp, P. Lasch, M. Beekes, L. Miller, and D. Naumann, "FTIR-microspectroscopy of prion-infected nervous tissue," Biochim. Biophys. Acta Biomembr. 1758, 948-959 (2006).

16. K. L. A. Chan and S. G. Kazarian, "Detection of trace materials with Fourier transform infrared spectroscopy using a multi-channel detector," Analyst (Cambridge, U.K.) 131, 126-131 (2006).

17. K. Golcuk, G. S. Mandair, A. F. Callender, N. Sahar, D. H. Kohn, and M. D. Morris, "Is photobleaching necessary for Raman imaging of bone tissue using a green laser?" Biochim. Biophys. Acta Biomembr. 1758, 868-873 (2006).

18. C. Krafft, L. Shapoval, S. B. Sobottka, K. D. Geiger, G. Schackert, and R. Salzer, "Identification of primary tumors of brain metastases by SIMCA classification of IR spectroscopic images," Biochim. Biophys. Acta Biomembr. 1758, 883-891 (2006).

19. N. Amharref, A. Beljebbar, S. Dukic, L. Venteo, L. Schneider, M. Pluot, R. Vistelle, and M. Manfait, "Brain tissue characterisation by infrared imaging in a rat glioma model," Biochim. Biophys. Acta Biomembr. 1758, 892-899 (2006).

20. K. R. Bambery, E. Schültke, B. R. Wood, S. T. R. MacDonald, K. Ataelmannan, R. W. Griebel, B. H. J. Juurlink, and D. McNaughton, "A Fourier transform infrared microspectroscopic imaging investigation into an animal model exhibiting glioblastoma multiforme," Biochim. Biophys. Acta Biomembr. 1758, 900-907 (2006).

21. S. Boydston-White, M. Romeo, T. Chernenko, A. Regina, M. Miljkovic, and M. Diem, "Cell-cycle-dependent variations in FTIR microspectra of single proliferating HeLa cells: Principal component and artificial neural network analysis," Biochim. Biophys. Acta Biomembr. 1758, 908-914 (2006)

22. A. L. Boskey, M. Goldberg, A. Kulkarni, and S. Gomez, "Infrared imaging microscopy of bone: Illustrations from a mouse model of Fabry disease," Biochim. Biophys. Acta Biomembr. 1758, 942-947 (2006)

23. K.-Z. Liu, A. Man, R. A. Shaw, B. Liang, Z. Xu, and Y. Gong, "Molecular determination of liver fibrosis by synchrotron infrared microspectroscopy," Biochim. Biophys. Acta Biomembr. 1758, 960967 (2006).

24. F. Bonnier, S. Rubin, L. Ventéo, C. M. Krishna, M. Pluot, B. Baehrel, M. Manfait, and G. D. Sockalingum, "In-vitro analysis of normal and aneurismal human ascending aortic tissues using FT-IR microspectroscopy," Biochim. Biophys. Acta Biomembr. 1758, 968-973 (2006).

25. M. K. Kuimova, K. L. A. Chan, and S. G. Kazarian, "Chemical imaging of live cancer cells in the natural aqueous environment," Appl. Spectrosc. 63, 164-171 (2009).

26. R. Mendelsohn, H.-C. Chen, M. E. Rerek, and D. J. Moore, "Infrared microspectroscopic imaging maps the spatial distribution of exogenous molecules in skin," J. Biomed. Opt. 8, 185-190 (2003).

27. C. Xiao, D. J. Moore, C. R. Flach, and R. Mendelsohn, "Permeation of dimyristoylphosphatidylcholine into skin-Structural and spatial information from IR and Raman microscopic imaging," Vib. Spectrosc. 38, 151 (2005). 
28. P. Garidel, "Insights in the biochemical composition of skin as investigated by micro infrared spectroscopic imaging," Phys. Chem. Chem. Phys. 5, 2673 (2003).

29. J. Jiang, M. Boese, P. Turner, and R. K. K. Wang, "Penetration kinetics of dimethyl sulphoxide and glycerol in dynamic optical clearing of porcine skin tissue in vitro studied by Fourier transform infrared spectroscopic imaging," J. Biomed. Opt. 13, 021105 (2008).

30. K. L. A. Chan and S. G. Kazarian, "Attenuated total reflection Fourier transform infrared imaging with variable angles of incidence: A three-dimensional profiling of heterogeneous materials," Appl. Spectrosc. 61, 48-54 (2007).

31. M. Boncheva, F. H. Tay, and S. G. Kazarian, "Application of attenuated total reflection Fourier transform infrared imaging and tapestripping to investigate the three-dimensional distribution of exogenous chemicals and the molecular organization in stratum corneum," J. Biomed. Opt. 13, 064009 (2008).

32. M. Krzic, M. Sentjurc, and J. Kristl, "Improved skin oxygenation after benzyl nicotinate application in different carriers as measured by EPR oximetry in vivo," J. Controlled Release 70, 203-211 (2001).

33. U. Jacobi, U. Erdmenger, M. Darvin, W. Sterry, and J. Lademann, "Determination of blood flow to study the penetration of benzyl nicotinate topically applied in different vehicles," Laser Phys. 16, 838841 (2006).
34. A. M. Kligman and E. Christophers, "Preparation of isolated sheets of human stratum corneum," Arch. Dermatol. 88, 702-705 (1963).

35. K. L. A. Chan, S. V. Hammond, and S. G. Kazarian, "Applications of attenuated total reflection infrared spectroscopic imaging to pharmaceutical formulations," Anal. Chem. 75, 2140-2147 (2003).

36. J. C. Tsai, "Tape stripping and sodium dodecyl sulfate treatment increase the molecular weight cutoff of polyethylene glycol penetration across murine skin," Arch. Dermatol. Res. 295, 169 (2003).

37. B. Berner, G. C. Mazzenga, J. H. Otte, R. J. Steffens, R. H. Juang, and C. D. Ebert, "Ethanol: Water mutually enhanced transdermal therapeutic system. II: Skin permeation of ethanol and nitroglycerin," J. Pharm. Sci. 78, 402-407 (1989).

38. A. C. Williams and B. W. Barry, "Penetration enhancers," Adv. Drug Delivery Rev. 56, 603-618 (2004).

39. J. L. V. dos Anjos, D. de Sousa Neto, and A. Alonso, "Effects of ethanol/l-menthol on the dynamics and partitioning of spin-labeled lipids in the stratum corneum," Eur. J. Pharm. Biopharm. 67, 406412 (2007)

40. C. Y. Goates and K. Knutson, "Enhanced permeation of polar compounds through human epidermis. I. Permeability and membrane structural changes in the presence of short chain alcohols," Biochim. Biophys. Acta Biomembr. 1195, 169-179 (1994). 